hautnah $2022 \cdot 21: 43-50$

https://doi.org/10.1007/s12326-022-00487-5

Angenommen: 14. Januar 2022

Online publiziert: 8. Februar 2022

(c) Der/die Autor(en) 2022

\title{
( )
}

\section{Epidemiologie und sozio- ökonomische Bedeutung}

Das Ulcus cruris venosum bezeichnet einen durch chronisch-venöse Insuffizienz (CVI) bedingten Substanzdefekt der unteren Extremität. Mit 45-60\% stellt es die häufigste Entität chronischer Ulzerationen der Beine dar [1]. Die Prävalenz für floride und abgeheilte venöse Ulzera liegt gemäß der Bonner Venenstudie bei $0,7 \%$ und bezüglich florider Ulzera bei $0,1 \%$. Mit höherem Alter steigt die Prävalenz allerdings deutlich an und beträgt in der Bevölkerungsgruppe der > 80-Jährigen $4-5 \%$. Vor dem 40 . Lebensjahr tritt das Ulcus cruris venosum (UCV) hingegen nur selten auf [2].

In einer alternden Gesellschaft stellen venöse Ulzera daher ein zunehmend relevantes Gesundheitsproblem dar, welches mit einer signifikanten Morbidität und einer hohen sozioökonomische Belastung verbunden ist [3]. Zu den Risikofaktoren, welche mit der Entstehung einer CVI assoziiert sind, zählen neben fortgestrittenem Alter auch das weibliche Geschlecht, eine positive Familienanamnese, Adipositas, multiple Schwangerschaften und vorrangig stehende oder sitzende Tätigkeiten [4-10].

\section{》) Die Behandlung ist} aufgrund der hohen Rate an Chronifizierung eine Herausforderung

Die Behandlung stellt vor allem aufgrund der hohen Rate an Chronifizierung eine Herausforderung dar [11-13]. Außerdem besteht eine hohe Rezidivrate von $30-70 \%$, wobei Patienten mit postthrombotischem Syndrom (PTS) häufi-

\section{B. Weber · J. Deinsberger}

Universitätsklinik für Dermatologie, Medizinische Universität Wien, Wien, Österreich

\section{Ulcus cruris venosum}

ger betroffen sind als jene Patienten, deren oberflächliche refluxive Gefäße interventionell behandelt wurden [14]. Risikofaktoren für eine protrahierte Wundheilung inkludieren eine große Wundfläche, eine längere Erkrankungsdauer und das Vorliegen einer Wundinfektion sowie fehlende Compliance der Patienten [15-17].

Der langwierige Heilungsprozess beeinträchtigt nicht nur signifikant die Lebensqualität der betroffenen Patienten, sondern führt auch zu einer substanziellen sozioökonomischen Belastung [18]. Schätzungen zufolge betragen die jährlichen Kosten für die Behandlung venöser Ulzera etwa $1 \%$ des gesamten Gesundheitsbudgets. $\mathrm{Zu}$ den direkten Gesundheitskosten für Behandlung und Spitalsaufenthalte entstehen auch indirekte Kosten durch Verminderung der Arbeitsfähigkeit [19]. Patienten mit UCV weisen außerdem, unabhängig von Alter und Geschlecht, eine erhöhte Langzeitsterblichkeit verglichen mit Kontrollgruppen auf [20].

\section{Pathophysiologie}

Die Entstehung eines Ulcus cruris venosum ist Zeichen einer weit fortgeschrittenen chronisch-venösen Insuffizienz. Diese ist durch eine Störung des venösen Flusses charakterisiert, die durch venösen Reflux, Obstruktion oder eine Kombination von beidem entsteht $[7,19,21]$.

Venöser Reflux entsteht insbesondere infolge inkompetenter Venenklappen und Dilatation der Venenwand und ist definiert durch eine Refluxdauer von $>0,5 \mathrm{~s}$ in superfiziellen Venen und $>1 \mathrm{~s}$ im subfaszialen Venensystem. Ein Reflux der superfiziellen Venen findet sich in bis zu $98 \%$ der Patienten mit venösem Ulkus und stellt in $70 \%$ die einzige $\mathrm{Pa}$ - thologie dar. Ein kombiniertes Auftreten von Reflux im oberflächlichen und tiefen Venensystem kann in etwa $12 \%$ der Fälle festgestellt werden. Die Angaben zu isoliert auftretendem Reflux in subfaszialen Venen schwanken zwischen 2 und $28 \%$, wobei bei etwa $30 \%$ dieser Patienten anamnestisch eine vorangegangene tiefe Venenthrombose besteht und somit ein postthrombotisches Syndrom diagnostiziert werden kann [22]. Die Rolle von refluxiven Perforansvenen in der Pathogenese der CVI bleibt umstritten [23].

Durch den venösen Rückfluss kommt es $\mathrm{zu}$ einer Druckerhöhung in den Stammvenen und in weiterer Folge auch in den venösen (post)kapillaren Gefäßen und somit zur Entwicklung einer venösen Hypertension. Davon unabhängig kann eine Dysfunktion der mikrovenösen Klappen auftreten und zu Reflux in kleinen oberflächlichen Venen und Kapillarschleifen führen, der sich in das mikrovenöse Netzwerk der Haut fortsetzen kann [24]. In weiterer Folge kommt es zu einem mikroangiopathischen Umbau, welcher mikroskopisch durch dilatierte Kapillaren höherer Tortuosität mit einem vergrößerten perikapillären Raum (Halo) charakterisiert ist [25].

Venöse Hypertension und Erweiterung der Venen resultieren in einer Verminderung der Scherspannung, was zur Aktivierung von Endothelzellen und in weiterer Folge zur Aktivierung und Transmigration von Leukozyten und zur Ausschüttung von proinflammatorischen Zytokinen, Chemokinen, Wachstumsfaktoren und Proteasen führt [26]. Weiters wird der Umbau der Glykokalyx der Endothelzellen initiiert. Im Zuge dessen entstehen alternierte Signalwege, und es kommt zu einem vermehrten $\mathrm{Ab}$ bau der Permeabiltätsbarriere, wodurch 
die Migration von Leukozyten und die Entzündungsreaktion weiter gefördert werden $[27,28]$.

Aufgrund der daraus resultierenden Hyperpermeabilität kommt es zur vermehrten Extravasation von proteinreicher Flüssigkeit und Erythrozyten in das Interstitium, wodurch die Bildung von Ödemen gefördert und eine adäquate Nährstoffversorgung des Gewebes beeinträchtigt wird [10, 29]. Dieser Prozess resultiert in einer anhaltenden Entzündungsreaktion sowie einer Störung der strukturellen Integrität der extrazellulären Matrix, was den Umbau der Venenwand und des umgebenden Gewebes zur Folge hat [30].

\section{》) Die entzündlichen \\ Gewebeveränderungen werden zunächst in Form einer Lipodermatosklerose sichtbar}

Eine vermehrte Produktion und veränderte Zusammensetzung von Kollagen (mehr Kollagen Typ I als Typ III) sowie die Verminderung von Elastin und Fibronektin führen zur Abnahme der Elastizität der Venenwand, was den vorhandenen Reflux weiter verschlechtert [27]. Die entzündlichen Veränderungen des Gewebes werden zunächst in Form einer Lipodermatosklerose und teilweise einer Atrophie blanche sichtbar. Kommt es in weiterer Folge zu einem kompletten Verlust der Epidermis sowie einer Schädigung des dermalen und subkutanen Gewebes, so wird ein Substanzdefekt in Form eines Ulkus sichtbar [4, 8, 9].

Die entstehenden Ulzerationen sind durch einen chronischen Fluss aus Entzündung und Gewebsumbau mit einer extensiven Zerstörung des Gewebes und Leukozyteninfiltraten gekennzeichnet, wodurch eine Heilung verhindert wird ([31]; • Abb. 1).

\section{Klinik und apparative Diagnostik}

UCV sind zumeist am unteren Drittel des Unterschenkels im Bereich des Innen- oder Außenknöchels lokalisiert und imponieren typischerweise als ent-

hautnah 2022 -21:43-50 https://doi.org/10.1007/s12326-022-00487-5

(c) Der/die Autor(en) 2022

\section{B. Weber ·. Deinsberger \\ Ulcus cruris venosum}

\section{Zusammenfassung}

Das Ulcus cruris venosum entsteht im Rahmen einer fortgeschrittenen chronischvenösen Insuffizienz und stellt die häufigste Entität chronischer Ulzerationen der unteren Extremität dar. Da die Prävalenz im Alter deutlich ansteigt, stellen venöse Ulzera in einer alternden Gesellschaft ein zunehmend relevantes Gesundheitsproblem dar, welches mit einer signifikanten Morbidität und einer hohen sozioökonomischen Belastung verbunden ist. Die Behandlung stellt aufgrund der niedrigen Heilungsrate, der häufigen Chronifizierung und der hohen Rezidivrate eine Herausforderung dar. Die Therapie umfasst in der Regel mehrere Modalitäten, wobei stets die Reduktion der venösen Hypertension in den zuführenden Gefäßen im Vordergrund steht. Bezüglich konservativer Therapieoptionen stehen die Kompressionstherapie, die pharmakologische Therapie sowie ergänzend die lokale Wundpflege zur Verfügung. Im Rahmen der kausalen, invasiven Therapie der dem Ulkus zugrunde liegenden Varkositas finden vor allem endoluminal-ablative Verfahren, die offene chirurgische Sanierung und die Schaumsklerotherapie ihre Anwendung. Neben einer Optimierung der invasiven Verfahren, liegt vor allem in der Entwicklung adjuvanter Therapiemodalitäten noch großes Potenzial.

Schlüsselwörter

Ulcus cruris venosum - Chronisch venöse Insuffizienz • Venöse Hypertension . Kompressionstherapie. Wundbehandlung

\section{Venous leg ulcers}

\section{Abstract}

Venous leg ulcers develop in the course of advanced chronic venous insufficiency and represent the leading cause for chronic ulcerations of the lower extremity. Since the prevalence increases with age, venous ulcers pose an increasingly relevant public health issue associated with significant morbidity of affected patients and a substantial socioeconomic burden. The treatment is challenging due to low healing rates, the chronic course of the disease and the high recurrence rate. Generally, the treatment includes multiple modalities aiming at the reduction of venous hypertension in affected vessels. Conservative therapeutic options include compression therapy, pharmacological therapy and complementary local wound care. Endoluminal ablative procedures, open surgical treatment and foam sclerotherapy represent curative, invasive treatment modalities aiming at the elimination of affected refluxive veins. Besides optimization of invasive treatment modalities, the development of adjuvant treatment options holds great potential for future improvements in the treatment of chronic venous ulcers.

\section{Keywords}

Venous leg ulcer - Chronic venous insufficiency · Venous hypertension - Compression therapy · Wound treatment zündlicher, schmerzhafter Substanzdefekt (• Abb. 2). Umfasst der Defekt den Unterschenkel zirkulär, so wird er als Gamaschenulkus bezeichnet. Im Anfangsstadium zeigt sich meist ein relativ flacher Defekt mit schmierig-eitrig belegtem Wundgrund. In der Wundumgebung zeigen sich oft auch weitere, durch die chronisch-venöse Insuffizienz hervorgerufene, Veränderungen, wie beispielsweise Hyperpigmentierungen (Purpura jaune docre), Atrophie blanche oder Dermato(lipofascio)sklerose.
Klinische Symptome, die mit chronisch-venöser Insuffizienz assoziiert sind, inkludieren ein Schweregefühl der Beine, Schmerzen und Unterschenkelödeme, welche mit einer signifikanten Beeinträchtigung der Lebensqualität der Patienten verbunden sind. Die Symptomatik wird typischerweise durch langes Stehen aggraviert. Mit fortschreitender Erkrankung entwickeln sich zunehmend auch die oben genannten trophischen Störungen der Haut sowie letztendlich schmerzhafte Ulzerationen [32-34]. 
Diagnostisch stehen die klinische Begutachtung und die Duplexsonographie im Vordergrund. Wegweisend ist hier die typische klinische Symptomatik verbunden mit den kutanen Veränderungen. Weiters werden die betroffenen Venen und der Grad der venösen Insuffizienz duplexsonographisch untersucht [35]. Grad und Ausprägung der CVI können mittels der CEAP(Clinical Etiology Anatomy Pathophysiology)-Klassifikation nachvollziehbar dokumentiert werden, wobei Grad C5 und C6 einem abgeheilten bzw. einem floriden Ulkus entsprechen [36]. Ergänzend sollten bei vorliegenden Entzündungszeichen Abstriche zur mikrobiologischen Testung entnommen werden. In Einzelfällen kann eine Gewebeentnahme zum Ausschluss eines Malignoms indiziert sein.

\section{》) Schweregefühl, Schmerzen} und Unterschenkelödeme sind klinische Symptome, die mit CVI assoziiert sind

Bei etwa $40 \%$ der Patienten besteht zudem eine Beeinträchtigung der arteriellen Durchblutung, die in zumindest $12 \%$ vorherrschend ist [21]. Die Feststellung einer arteriellen Makroperfusionsstörung ist insbesondere vor einer Kompressionstherapie relevant. Daher sollte die Basisdiagnostik eines UCV stets auch die Bestimmung des Knöchel-Arm-Index beinhalten.

\section{Konservative Therapieoptionen}

Die Therapie des UCV umfasst in der Regel mehrere Modalitäten, wobei stets die Reduktion der venösen Hypertension im Ulkusbereich im Vordergrund steht. Als konservative Therapieoptionen stehen hierfür die Kompressionstherapie und die pharmakologische Therapie zur Verfügung. Ergänzend stellen auch die lokale Wundpflege und -dokumentation einen wichtigen Eckpfeiler der konservativen UCV-Therapie dar.

\section{Kompressionstherapie}

Die Kompressionstherapie nimmt im Rahmen der Behandlung des UCV eine zentrale Rolle ein und stellt die wichtigste konservative Therapiemodalität dar. Sie reduziert die venöse Hypertension und verbessert den venösen Rückfluss, wodurch die Ausbildung von Phlebödemen hintangehalten wird. Zahlreiche Studien konnten zeigen, dass die Kompressionstherapie sowohl $\mathrm{zu}$ einer allgemein höheren Abheilungsrate als auch zu einer höheren Abheilungsgeschwindigkeit eines UCV führt. Außerdem konnte gezeigt werden, dass die assoziierten Schmerzen signifikant verringert werden und auch die Lebensqualität bezogen auf die UCV substanziell verbessert wird [37].

\section{》) Die Kompressionstherapie} reduziert die venöse Hypertension und verbessert den venösen Rückfluss

Die Verwendung von Mehrkomponentensystemen zeigte hierbei eine höhere Effektivität im Vergleich zu EinzelKomponenten-Systemen. Während früher Kompressionsverbände als Erstlinientherapie favorisiert wurden, haben rezente Untersuchungen eine Überlegenheit medizinischer Kompressionsstrümpfe gezeigt. Diese führten sowohl zu einer höheren Abheilungsrate als auch -geschwindigkeit [38].

Außerdem erscheint der deutlich verbesserte Tragekomfort und damit eine optimale Therapiecompliance ein wesentlicher Vorteil von Kompressionsstrumpf-Systemen zu sein [39]. Ein weiterer wesentlicher Vorteil besteht in der konstanten Aufrechterhaltung von Kompressionsdrücken im Vergleich zu Bandagen [40]. Neben einer verbesserten Abheilungsrate führte die Anwendung medizinischer Kompressionsstrümpfe $\mathrm{zu}$ einer signifikanten Abnahme von Ulkusrezidiven [41].

Im Rahmen der ESCHAR-Studie zeigte sich außerdem, dass durch eine Kombination von Kompressionstherapie mit chirurgischer Therapie der zu
Hier steht eine Anzeige. Springer 


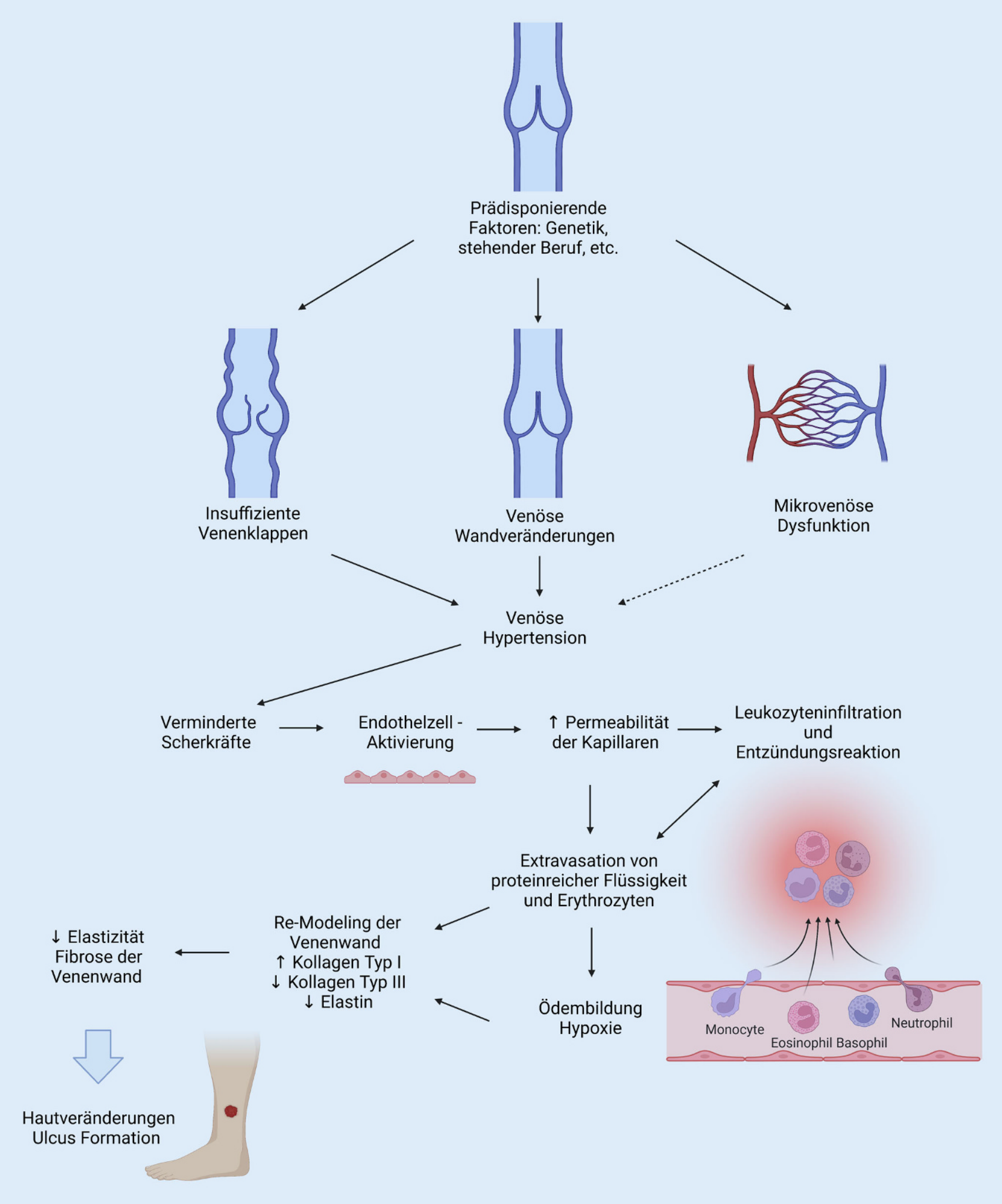

Abb. 1 A Pathogenese des Ulcus cruris venosum. (Diese Abbildung wurde mithilfe von biorender.com erstellt) 

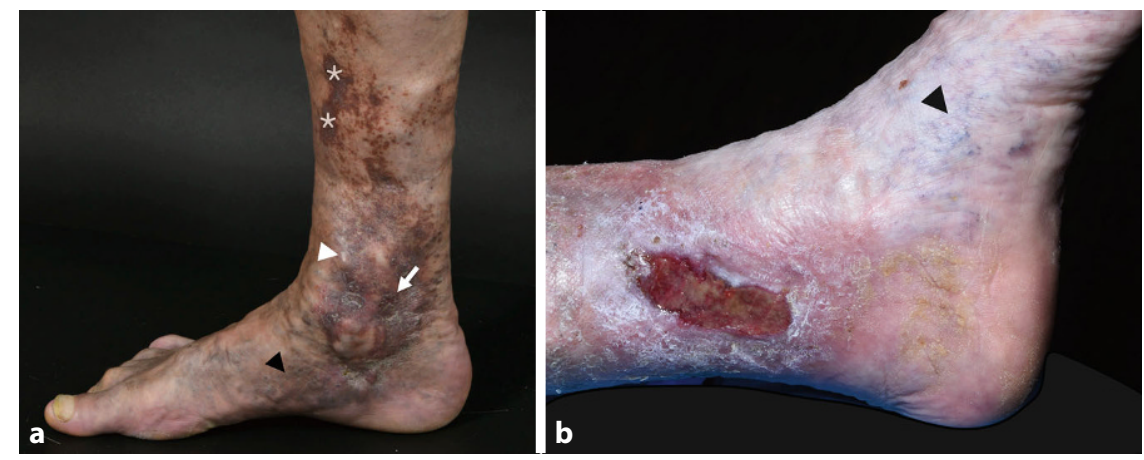

Abb. $2 \Delta$ a Fortgeschrittene chronisch-venöse Insuffizienz bei einem 50-jährigen Patienten. WeiBer Pfeil abgeheiltes Ulkus (Stadium C5 der CEAP-Klassifikation), weiße Pfeilspitze Atrophie blanche, schwarze Pfeilspitze Corona phlebectatica paraplantaris, Sterne Hyperpigmentierung (Purpura jaune d'ocre). b Ulcus cruris (Stadium C6 der CEAP-Klassifikation) im Bereich des medialen Knöchels bei einer 77-jährigen Patientin. Schwarze Pfeilspitze Corona phlebectatica paraplantaris

Grunde liegenden Varikositas die Rezidivrate von UCV weiter gesenkt werden konnte [42]. Es zeigte sich, dass erhöhte Kompressionsdrücke (25-35 mm Hg, Klasse II) gegenüber niedrigeren Werten (18-24 mm Hg, Klasse I) die Effektivität weiter erhöhen und die Rezidivrate weiter senken $[13,43]$. Hierbei gilt es jedoch zu beachten, dass die Compliance der Patienten mit zunehmenden Kompressionsdrücken entsprechend abnimmt.

Im klinischen Alltag gilt es daher auch auf diesen Aspekt Rücksicht zu nehmen und eine individuelle Therapie für den Patienten vorzusehen. Im Einzelfall ist einer Therapie mit geringerem Kompressionsdruck der Vorzug zu geben, wenn absehbar ist, dass eine Therapie mit höheren Kompressionsdrücken ohnehin in einer Malcompliance mündet. Ein möglicher Lösungsansatz stellt die Anwendung von Zweilagensystemen dar. Hierbei wird ein Unterstrumpf mit Druckwerten im Bereich der Kompressionsklasse I (18-24 mm Hg) mit einem Überstrumpf der Kompressionsklasse II (23-32 mm Hg) kombiniert. Durch die Kombination dieser zwei Strumpfsysteme können insgesamt Druckwerte von bis $\mathrm{zu} 40 \mathrm{~mm} \mathrm{Hg}$ erreicht werden, wodurch eine weitere Optimierung der Abheilungsrate von UCV erzielt werden kann. Außerdem kann das Anlegen erleichtert werden und die physikalischen Eigenschaften („stiffness“) optimiert werden [44]. Bezüglich der Anwendung der intermittierenden pneumatischen Kompression zur Therapie des UCV liegt derzeit keine ausreichende Evidenz vor, um dessen routinemäßigen Einsatz zu rechtfertigen.

Im Rahmen der Kompressionstherapie gilt es $\mathrm{zu}$ beachten, dass eine ausreichende arterielle Versorgung der Extremität eine Grundvoraussetzung darstellt. Sollte trotz insuffizienter arterieller Versorgung eine Kompressionstherapie erfolgen, so kann dies zu ischämischem Gewebeuntergang und Hautnekrosen führen. Insbesondere bei Patienten mit kritischer Extremitätenischämie, systolischen Druckwerten im Knöchelbereich von $<70 \mathrm{~mm} \mathrm{Hg}$ oder nach erfolgter Bypassanlage am Bein ist eine Kompressionstherapie kontraindiziert [45].

\section{Pharmakologische Therapie}

Obwohl die Kompressionstherapie gewissermaßen den Grundpfeiler einer jeden UCV-Therapie darstellt und vor allem die Rezidivrate deutlich beeinflussen kann, so zeigten einige Studien, dass der Effekt der Kompressionstherapie auf die Abheilung bereits bestehender Ulzera begrenzt ist. Nach zwei Jahren Kompressionstherapie waren teils noch immer bis zu $50 \%$ der Ulzera nicht vollständig abgeheilt [46].

Die Gründe hierfür sind wahrscheinlich multifaktoriell. Neben einer suboptimalen Compliance werden vor allem chronische Entzündungsprozesse im Rahmen der CVI als Ursachen genannt. Aus diesem Grund haben zahlreiche Studien den zusätzlichen Nutzen einer adjuvanten Pharmakotherapie von
UCV untersucht. Die verschiedenen derzeit verfügbaren venoaktiven Pharmaka setzen vorwiegend im Bereich der verbesserten Mikrozirkulation und reduzierten Inflammation an [47]. $\mathrm{Zu}$ den wichtigsten Vertretern zählen hierbei Flavonoide, Pentoxifyllin, Sulodexid und Mesoglycan.

》) Der Effekt der Kompressionstherapie auf die Abheilung bereits bestehender Ulzera ist begrenzt

Die Gruppe der Flavonoide stellt derzeit wohl die prominenteste Gruppe an Therapeutika dar. Ihre Wirksamkeit bei UCV wurde in zahlreichen Studien untersucht. Unter dem Überbegriff der Flavonoide werden verschiedene Substanzen, wie die mikronisiert purifizierte Flavonoidfraktion („MPFF“), Diosmin oder Hidrosmin zusammengefasst. Die Wirkung von Flavonoiden basiert hierbei auf mehreren Ansatzpunkten. Zum einen weisen diese direkt vasoaktiv-venotonische Eigenschaften auf. Zum anderen reduzieren sie die lokale Entzündung und EndothelLeukozyten-Interaktion, wodurch auch die Permeabilität der Mikrozirkulation und damit die Inflammation positiv beeinflusst werden [48].

Während einige Studien keine signifikanten Effekte fanden, zeigten sich in anderen eine deutlich raschere Abheilung von Ulzera. Sowohl in der Übersichtsarbeit von Coleridge-Smith et al. [49] zu MPFF als auch im CochraineReview von Martinez et al. [50] wurden die bislang publizierten Studien analysiert, wobei insgesamt jeweils eine signifikant raschere Abheilung unter einer Therapie mit Flavonoiden allgemein bzw. mit MPFF im Einzelnen festgesellt wurde. Interessanterweise zeigte sich in der Metaanalyse von Coleridge-Smith et al. [49], dass ein signifikanter Effekt auf die Abheilungsrate erst bei länger bestehenden Ulzera ( $>6$ Monate) bestand. Auch in der Metaanalyse von Jantet et al. zeigte sich eine um insgesamt $37 \%$ erhöhte Abheilungsrate von UCV unter Therapie mit MPFF [51]. 
Neben der prominenten Stoffgruppe der Flavonoide konnten unter anderem auch für die Wirkstoffe Pentoxifyllin, Sulodexid, and Mesoglycan positive Effekte auf die Abheilungsrate und -geschwindigkeit von UCV festgestellt werden [47]. Hierbei gilt es jedoch zu betonen, dass die Pharmakotherapie jeweils nur eine adjuvante Therapieoption zusätzlich zu weiteren Therapiemodalitäten darstellt und die kausale Therapie von UCV im Mittelpunkt der therapeutischen Maßnahmen stehen sollte.

\section{Lokale Wundtherapie}

Neben der Größe und Bestandsdauer einer Wunde ist das Vorhandensein einer Superinfektion ein wesentlicher Aspekt, welcher die Wundheilung allgemein negativ beeinflussen kann. Deshalb wird im Rahmen der Wundbehandlung eine regelmäßige Reinigung der Ulzera empfohlen. Dies wird in der Regel mit physiologischer Kochsalzlösung durchgeführt. Weiters können auch wasserbasierte, nichtzytotoxische Lösungen, beispielsweise basierend auf hypochloriger Säure, verwendet werden [52].

Sollten im Rahmen der Heimtherapie keine derartigen Lösungen zur Verfügung stehen, so kann, bei entsprechender Wasserqualität, auch auf Leitungswasser zurückgegriffen werden [44]. Im Cochrane-Review von Fernandez et al. [53] zeigte sich keine Evidenz für erhöhte Infektionsraten unter dieser Therapie. Sollten sich klinische Zeichen einer Wundinfektion ergeben, so sollte eine Behandlung mit lokalen Antiseptika erfolgen, wobei bei chronischen Wunden vor allem Antiseptika auf Basis von Octenidine Dihydrochlorid oder Polihexanid empfohlen werden [54, 55].

Für die lokale Behandlung von UCV wird eine Vielzahl an verschiedenen Wundauflagen und -materialien verwendet. Dies schließt beispielsweise Hydrokolloide, Hydrogele, Folien, Hydrofasern, Laminate, Polyurethanschaumstoffe, silberhaltige Externa oder medizinischen Honig ein. Wie im Cochrane-Review von Norman et al. augenscheinlich dargelegt wird, besteht hierbei jedoch bislang ein ausgesprochener Mangel an solider wissenschaftlicher Evidenz, in Form von kontrollierten Studien [56].

Bis zum Vorliegen solider wissenschaftlicher Evidenz sollte daher vor allem der Patientenkomfort im Vordergrund von Therapieüberlegungen stehen und auf allgemeine Prinzipien der Wundtherapie, wie die feuchte Wundbehandlung, geachtet werden. Zur weiteren Unterstützung der Abheilung werden teils auch Vakuumtherapien („negative pressure wound therapy“) zur Unterstützung der Abheilung und Reduktion von Mikroorganismen eingesetzt. Doch auch hier fehlt es derzeit noch an solider wissenschaftlicher Evidenz [57], um einen generellen Einsatz der Vakuumtherapie bei UCV zu empfehlen. Vielmehr zeigte eine randomisierte Studie von Leclercq et al., dass die Vakuumtherapie nach Spalthauttransplantation bei Beinulzera sogar mit einer erhöhten Komplikationsrate vergesellschaftet war [58]. Diese Ergebnisse machen augenscheinlich, dass die Lokaltherapie von UCV bzw. von Beinulzera allgemein auf wenig wissenschaftlicher Evidenz basiert und zukünftige, kontrollierte Studien dringend erforderlich sind.

\section{Interventionelle Therapieoptionen}

Das Ziel einer jeden interventionellen Therapie der Varikose allgemein und des UCV im Speziellen ist die Ausschaltung der pathologischen venösen Hypertension. Aufgrund der Tatsache, dass dieser Ansatz eine kausale Behandlung darstellt, ist eine zeitnahe Sanierung einer bestehenden superfiziellen venösen Insuffizienz anzustreben. Hierfür stehen heutzutage zahlreiche therapeutische Modalitäten zur Verfügung, weshalb die Therapie individuell auf die Bedürfnisse der jeweiligen Patienten abgestimmt werden kann.

Im Rahmen der ESCHAR-Studie [59] konnte gezeigt werden, dass ein invasiv-chirurgisches Vorgehen, im Sinne einer Crossektomie kombiniert mit Venenstripping und einer Kompressionstherapie, zu einer signifikanten Reduktion an Ulkusrezidiven nach 1 bzw. 4 Jahren führt. Auf Basis dieser und weiterer Studien setzte somit ein Umdenken hin zu einer proaktiven, chirurgischen Therapie von UCV ein.

In den darauffolgenden Jahren lösten jedoch die endoluminal-thermisch-ablativen Verfahren, die endovenöse Laserablation (EVLA) und die radiofrequenzbasierte Ablation die offen-chirurgische Sanierung der Varikositas sukzessive ab [60]. Dies fand auch entsprechend Anwendung für die Therapie von UCV, wo sich in zahlreichen Studien vergleichbare Abheilungs- und Rezidivraten in Bezug auf offen-chirurgische Verfahren zeigten $[60,61]$.

Im Rahmen der randomisierten Studie von Gohel et al. zeigte sich vor allem die Wichtigkeit einer frühen interventionellen Therapie, wodurch eine raschere Abheilung und auch eine Kostenersparnis in der Ulkusbehandlung erzielt werden kann [61]. Neben den thermischablativen Verfahren wurde zuletzt auch vermehrt über die Anwendung von Cyanoacrylat-Kleber zur Refluxausschaltung bei UCV-Patienten berichtet. In einer rezenten Arbeit von O'Banion et al. wurde sogar über eine Überlegenheit dieser Methode im Vergleich zur thermischen Ablation in Bezug die Dauer der Abheilungszeit berichtet [62].

Neben den operativen bzw. interventionell-invasiven Verfahren stellt die Schaumverödungstherapie einen wichtigen Grundpfeiler der Therapie von UCV dar. Auch wenn die Schaumverödungstherapie im Allgemeinen den interventionellen und chirurgischen Therapien der Varikositas in Bezug auf die Häufigkeit von Rezidiven unterlegen ist [63], so nimmt sie doch im Rahmen der UCVTherapie eine besondere Stellung ein.

Zum einen sind die invasiven Therapien für die meist ältere Patientenpopulation oftmals aufgrund von Begleiterkrankungen nicht anwendbar, weshalb die Schaumverödungstherapie hier eine versatile wie auch wirksame Therapiealternative darstellt [64]. Zum anderen können im Rahmen der Schaumsklerotherapie auch insuffiziente Kleinstgefäße direkt unterhalb des Ulkus mitbehandelt werden [65]. Dadurch wird gewissermaßen eine Abkoppelung der Ulkusumgebung von der makrovaskulären venösen Hypertension erzielt, was beispielsweise bei endoluminal-thermischen Verfahren 
nicht der Fall ist. Dies erklärt den besonderen Stellenwert der Schaumsklerotherapie im Rahmen der Therapie des UCV, was auch in zahlreichen Studien nachgewiesen werden konnte [64].

Die Sklerotherapie, ob mit $\mathrm{Mi}$ kroschaum oder ohne, kann ferner auch mit invasiven katheterbasierten Verfahren kombiniert werden, was als mechanochemische Ablation (MOCA) bezeichnet wird. Auch hier berichten erste Studien vergleichbare Resultate in Bezug auf endoluminal-thermische Verfahren [66]. Insbesondere die fehlende Notwendigkeit einer Tumeszenzlokalanästhesie mit Zusätzen von Epinephrin und Lidocain macht die MOCA-Methode, vor allem bei kardial vorbelasteten Patienten mit UCV, besonders attraktiv. Aufgrund dieser Fülle an Therapiemodalitäten gilt es daher, einen individuell auf den Patienten zugeschnittenen Therapieplan $\mathrm{zu}$ erstellen, um eine rasche und nachhaltige Abheilung von UCV zu erreichen. Hierbei kann auch eine Kombination an interventionellen bzw. venenchirurgischen Verfahren, Schaumsklerotherapie oder auch einer Spalthauttransplantation im Rahmen der gleichen Sitzung erfolgen.

\section{Kommentar und Ausblick}

Das Ulcus cruris venosum ist eine Herausforderung: für betroffene Patienten, deren Lebensqualität dadurch stark eingeschränkt ist, für globale Gesundheitssysteme, wo Milliarden in die aufwändige Behandlung dieser Erkrankung investiert werden, aber auch für das medizinische Personal, welches sich mit dieser hochchronischen Erkrankung auseinandersetzt.

Durch die Entwicklungen der letzten zwei Jahrzehnte stehen uns immer bessere und vor allem weniger invasive Behandlungsmethoden zur Verfügung, um eine rasche und nachhaltige Abheilung der Ulzera zu erzielen. Trotzdem kann eine Abheilung nicht in jedem Fall erreicht werden, und Ulkusrezidive stehen weiterhin an der Tagesordnung.

Neben einer Optimierung der invasiven Verfahren liegt vor allem in der Entwicklung adjuvanter Therapiemodalitäten noch großes Potenzial. Dies in- kludiert unter anderem folgende Technologien: elektrische Stimulation, Ultraschalltherapie, elektromagnetische Therapie, Photobiomodulation, Sauerstofftherapie, Platelet-Rich-Plasma-Therapie, Biologika oder Zelltherapie [67]. Ob diese Therapien tatsächlich einen relevanten Effekt beim UCV haben, werden erst zukünftige Untersuchungen zeigen. Es bleibt zu hoffen, dass über diese Entwicklungen eine weitere Verbesserung der Therapie dieser häufigen Erkrankung erzielt werden kann.

\section{Korrespondenzadresse}

\section{B. Weber}

Universitätsklinik für Dermatologie,

Medizinische Universität Wien

Währinger Gürtel 18-20, 1090 Wien, Österreich

benedikt.weber@meduniwien.ac.at

Funding. Open access funding provided by Medical University of Vienna.

\section{Einhaltung ethischer Richtlinien}

Interessenkonflikt. B. Weber und J. Deinsberger geben an, dass kein Interessenkonflikt besteht.

Für diesen Beitrag wurden von den Autoren keine Studien an Menschen oder Tieren durchgeführt. Für die aufgeführten Studien gelten die jeweils dort angegebenen ethischen Richtlinien.

Open Access. Dieser Artikel wird unter der Creative Commons Namensnennung 4.0 International Lizenz veröffentlicht, welche die Nutzung, Vervielfältigung, Bearbeitung, Verbreitung und Wiedergabe in jeglichem Medium und Format erlaubt, sofern Sie den/die ursprünglichen Autor(en) und die Quelle ordnungsgemäß nennen, einen Link zur Creative Commons Lizenz beifügen und angeben, ob Änderungen vorgenommen wurden.

Die in diesem Artikel enthaltenen Bilder und sonstiges Drittmaterial unterliegen ebenfalls der genannten Creative Commons Lizenz, sofern sich aus der Abbildungslegende nichts anderes ergibt. Sofern das betreffende Material nicht unter der genannten Creative Commons Lizenz steht und die betreffende Handlung nicht nach gesetzlichen Vorschriften erlaubt ist, ist für die oben aufgeführten Weiterverwendungen des Materials die Einwilligung des jeweiligen Rechteinhabers einzuholen.

Weitere Details zur Lizenz entnehmen Sie bitte der Lizenzinformation auf http://creativecommons.org/ licenses/by/4.0/deed.de.

\section{Literatur}

1. Mekkes JR et al (2003) Causes, investigation and treatment of leg ulceration. Br J Dermatol 148(3):388-401

2. Rabe $E$, Pannier-Fischer $F$, Bromen $K$, Schuldt $K$, Stang A, Poncar C et al (2003) Bonn Vein Study by the German Society of Phlebology: Epidemiologicalstudy to investigate the prevalence and severity of chronic venous disorders in the urban and rural residential populations. Phlebologie 32:1-14

3. Duschek N, Trautinger F (2019) Chronic leg ulcers in older patients. Z Gerontol Geriatr 52(4):377-390

4. Bonkemeyer Millan S, Gan R, Townsend PE (2019) Venous ulcers: diagnosis and treatment. Am Fam Physician 100(5):298-305

5. Phillips TJ, Dover JS (1991) Leg ulcers. J Am Acad Dermatol 25(6 Pt 1):965-987

6. Xie T et al (2018) The venous ulcer continues to be a clinical challenge: an update. Burn Trauma 6:18-18

7. Nicolaides AN (2000) Investigation of chronic venous insufficiency: a consensus statement (France, march 5-9, 1997). Circulation 102(20):E126-E163

8. van Gent WB, Wilschut ED, Wittens C (2010) Management of venous ulcer disease. BMJ 341:c6045

9. Bollinger A et al (1997) Microvascular changes in venous disease: an update. Angiology 48(1):27-32

10. Eberhardt RT, Raffetto JD (2014) Chronic venous insufficiency. Circulation 130(4):333-346

11. Finlayson KJ et al (2018) Predicting the likelihood of venous leg ulcer recurrence: The diagnostic accuracy of a newly developed risk assessment tool. Int Wound J 15(5):686-694

12. McDaniel HB et al (2002) Recurrence of chronic venous ulcers on the basis of clinical, etiologic anatomic, and pathophysiologic criteria and air plethysmography. J Vasc Surg 35(4):723-728

13. Nelson EA et al (2006) Prevention of recurrence of venous ulceration: Randomized controlled trial of class 2 and class 3 elastic compression. J Vasc Surg 44(4):803-808

14. Fritsch P (2004) Dermatologie Venerologie: Grundlagen. Klinik. Atlas. Springer, Berlin-Heidelberg

15. Margolis DJ, Berlin JA, Strom BL (1999) Risk factors associated with the failure of a venous leg ulcer to heal. Arch Dermatol 135(8):920-926

16. Gohel MS et al (2005) Risk factors for delayed healing and recurrence of chronic venous leg ulcers-an analysis of 1324 legs. Eur J Vasc Endovascular Surg 29(1):74-77

17. Deol ZK et al (2020) Effect of obesity on chronic venous insufficiency treatment outcomes. J Vasc Surg Venous Lymphat Disord 8(4):617-628.e1

18. Chi YW, Raffetto JD (2015) Venous leg ulceration pathophysiology and evidence based treatment. Vasc Med. https://doi.org/10.1177/ $1358863 \times 14568677$

19. Ma H et al (2014) The real cost of treating venous ulcers in a contemporary vascular practice. J Vasc Surg Venous Lymphat Disord 2(4):355-361

20. Salenius JEetal (2021) Long-term Mortalityamong Patients with Chronic Ulcers. Acta Derm Venereol 101(5):adv455

21. Nelzén O, Bergqvist D, Lindhagen A (1991) Leg ulcer etiology - a cross sectional population study. JVasc Surg 14(4):557-564

22. Kanth AM et al (2015) The distribution and extent of reflux and obstruction in patients with active venous ulceration. Phlebology 30(5):350-356

23. Cavezzi A (2020) Medicineand phlebolymphology: Time to change? JClin Med 9(12):4091. https://doi. org/10.3390/jcm 9124091 
24. Vincent JR et al (2011) Failure of microvenous valves in small superficial veins is a key to the skin changes of venous insufficiency. J Vasc Surg 54(6 Suppl):62S-69S.e1-3

25. Howlader MH, Smith PD (2003) Microangiopathy in chronic venous insufficiency: quantitative assessment by capillary microscopy. Eur J Vasc Endovasc Surg 26(3):325-331

26. Castro-Ferreira Retal (2018) The role of endothelia dysfunction and inflammation in chronic venous disease. Ann Vasc Surg 46:380-393

27. Ortega MA et al (2021) Understanding chronic venous disease: A critical overview of its pathophysiology and medical management. J Clin Med 10(15):3239. https://doi.org/10.3390/ jcm10153239

28. Tarbell JM, Cancel LM (2016) The glycocalyx and its significance in human medicine. J Intern Med 280(1):97-113

29. Raffetto JD (2016) Pathophysiology of wound healing and alterations in venous leg ulcersreview. Phlebology 31(1 Suppl):56-62

30. Raffetto JD, Mannello F (2014) Pathophysiology of chronic venous disease. Int Angiol 33(3):212-221

31. Parker TJ et al (2015) Provisional matrix deposition in hemostasis and venous insufficiency: tissue preconditioning for Nonhealing venous ulcers. Adv Wound Care 4(3):174-191

32. Santler B, Goerge T (2017) Chronic venous insufficiency - a review of pathophysiology, diagnosis, and treatment. J Dtsch Dermatol Ges 15(5):538-556. https://doi.org/10.1111/ddg. 13242

33. Vivas A, Lev-Tov H, Kirsner RS (2016) Venous leg ulcers. Ann Intern Med 165(3):ITC17-ITC32

34. Green J et al (2014) The impact of chronic venous leg ulcers: a systematic review. J Wound Care 23(12):601-612

35. Gloviczki P et al (2011) The care of patients with varicose veins and associated chronic venous diseases: clinical practice guidelines of the Society for Vascular Surgery and the American Venous Forum. J Vasc Surg 53(5 Suppl):2s-48s

36. Lurie $F$ et al (2020) The 2020 update of the CEAP classification system and reporting standards. J Vasc Surg Venous Lymphat Disord 8(3):342-352. https://doi.org/10.1016/j.jvsv.2019.12.075. Erratum in: J Vasc Surg Venous Lymphat Disord 9(1):288

37. Shi $C$ et al (2021) Compression bandages or stockings versus no compression for treating venous leg ulcers. Cochrane Database Syst Rev. https://doi.org/10.1002/14651858.CD013397

38. Stücker M et al (2013) Compression and venous ulcers. Phlebology 28(Suppl 1):68-72

39. AmslerF, Willenberg T, BlättlerW (2009) In search of optimal compression therapy forvenous legulcers: a meta-analysis of studies comparing diverse [corrected] bandages with specifically designed stockings. JVasc Surg 50(3):668-674

40. Ghosh $S$ et al (2008) Pressure mapping and performance of the compression bandage/ garment for venous leg ulcer treatment. J Tissue Viability 17(3):82-94

41. O'Meara S et al (2012) Compression for venous leg ulcers. Cochrane Database Syst Rev. https://doi. org/10.1002/14651858.CD000265.pub3

42. Gohel MS, Barwell JR, Taylor M, Chant T, Foy C, Earnshaw JJ et al (2007) Long term results of compression therapy alone versus compression plus surgery in chronic venous ulceration (ESCHAR) randomised controlled trial. BMJ 335(7610):83. https://doi.org/10.1136/bmj.39216.542442.BE
43. Milic DJ et al (2010) The influence of different subbandage pressure values on venous leg ulcers healing when treated with compression therapy. JVasc Surg 51(3):655-661

44. Böhler K (2016) Das venöse Ulcus cruris. Wien Med Wochenschr 166(9):287-292

45. RabeEetal (2018) Indicationsformedical compression stockings in venous and lymphatic disorders: an evidence-based consensus statement. Phlebology 33(3):163-184

46. de Oliveira Carvalho PE et al (2016) Oral aspirin for treating venous leg ulcers. Cochrane Database Syst Rev. https://doi.org/10.1002/14651858. CD009432.pub2

47. Kitchens BP, Snyder RJ, Cuffy CA (2020) A literature review of pharmacological agents to improve venous leg ulcer healing. Wounds 32(7):195-207

48. Pascarella L, Schönbein GW, Bergan JJ (2005) Microcirculation and venous ulcers: a review. Ann Vasc Surg 19(6):921-927

49. Coleridge-Smith P, Lok C, Ramelet AA (2005) Venous leg ulcer: a meta-analysis of adjunctive therapy with micronized purified flavonoid fraction. Eur JVasc Endovasc Surg 30(2):198-208

50. Martinez-Zapata MJ et al (2016) Phlebotonics for venous insufficiency. Cochrane Database Syst Rev. https://doi.org/10.1002/14651858.CD003229. pub4

51. Jantet G (2002) Chronic venous insufficiency: worldwide results of the RELIEF study. Reflux assEssment and quaLity of Ilfe improvEment with micronized Flavonoids. Angiology 53(3):245-256

52. Bongiovanni CM (2014) Effects of hypochlorous acid solutions on venous leg ulcers (VLU): experience with 1249 VLus in 897 patients. J Am Coll Clin Wound Spec 6(3):32-37

53. Fernandez R, Griffiths R (2012) Water for wound cleansing. Cochrane Database Syst Rev. https:// doi.org/10.1002/14651858.CD003861.pub3

54. Bellingeri $A$ et al (2016) Effect of a wound cleansing solution on wound bed preparation and inflammation in chronic wounds: a single-blind RCT. JWound Care 25(3):160-162-6, 168

55. Daeschlein G (2013) Antimicrobial and antiseptic strategies in wound management. Int Wound J 10(Suppl 1):9-14

56. Norman $\mathrm{G}$ et al (2018) Dressings and topical agents for treating venous leg ulcers. Cochrane Database Syst Rev. https://doi.org/10.1002/ 14651858.CD012583.pub2

57. Dumville JC et al (2015) Negative pressure wound therapy for treating leg ulcers. Cochrane Database Syst Rev. https://doi.org/10.1002/14651858. CD011354.pub2

58. Leclercq A et al (2016) Skin graft secured by VAC (vacuum-assisted closure) therapy in chronic leg ulcers: A controlled randomized study. Ann Dermatol Venereol 143(1):3-8

59. Barwell JR et al (2004) Comparison of surgery and compression with compression alone in chronic venous ulceration (ESCHAR study): randomised controlled trial. Lancet 363(9424):1854-1859

60. Marston WA et al (2017) Incidence of venous leg ulcer healing and recurrence after treatment with endovenous laser ablation. J Vasc Surg Venous Lymphat Disord 5(4):525-532

61. Gohel MS et al (2020) Long-term clinical and cost-effectiveness of early endovenous ablation in venous ulceration: a randomized clinical trial. JAMA Surg 155(12):1113-1121

62. O'Banion LA et al (2021) A comparison of cyanoacrylate glue and radiofrequency ablation techniques in the treatment of superficial venous reflux in CEAP 6 patients. J Vasc Surg Venous Lymphat Disord 9(5):1215-1221

63. Lawaetz M et al (2017) Comparison of endovenous ablation techniques, foam sclerotherapy and surgical stripping for great saphenous varicose veins. Extended 5-year follow-up of a RCT. Int Angiol 36(3):281-288

64. Kahle B (2017) Spezifische Indikationen zur Schaumsklerosierung. Hautarzt 68(8):621-624

65. Lloret P et al (2015) Treatment of venous leg ulcers with ultrasound-guided foam sclerotherapy: Healing, long-term recurrence and quality of life evaluation. Wound Repair Regen 23(3):369-378

66. Kim SY et al (2019) Mechanochemical ablation as an alternative to venous ulcer healing compared with thermal ablation. J Vasc Surg Venous Lymphat Disord 7(5):699-705

67. Aleksandrowicz H, Owczarczyk-Saczonek A, PlacekW(2021) Venouslegulcers:advanced therapies and new technologies. Biomedicines 9(11):1569. https://doi.org/10.3390/biomedicines9111569

Hinweis des Verlags. Der Verlag bleibt in Hinblick auf geografische Zuordnungen und Gebietsbezeichnungen in veröffentlichten Karten und Institutsadressen neutral. 